\title{
Monitoring Acute Diarrhea via an Electronic Surveillance System in the Peruvian Navy
}

\author{
Emily Alsentzer*1, Delphis M. Vera², Joan Neyra ${ }^{3}$, Luis Loayza7, Ricardo A. Hora², Victor B. \\ Osorio $^{5}$, Jose Quispe ${ }^{6}$, Sarah-Blythe Ballard ${ }^{4}$ and David Blazes ${ }^{3}$
}

'Stanford University, Stanford, CA, USA; ${ }^{2}$ Naval Medical Research Unit No. Six, Lima, Peru; ${ }^{3}$ Uniformed Services University of the Health Sciences, Bethesda, MD, USA; ${ }^{4}$ Johns Hopkins Bloomberg School of Public Health, Baltimore, MD, USA; ${ }^{5}$ Universidad Nacional Agraria La Molina, Lima, Peru; ${ }^{6}$ Dirección de Salud de la Marina de Guerra del Peru, Lima, Peru; ${ }^{7}$ Marina de Guerra del Perú, Lima, Peru

\section{Objective}

To use data from the Peruvian Navy's electronic syndromic surveillance systems to estimate the baseline incidence of acute diarrheal disease (ADD) and detect outbreaks among individuals accessing military medical facilities from 2009-13.

\section{Introduction}

Syndromic surveillance can supplement diagnosis-based surveillance in resource-limited settings with limited laboratory infrastructure. Syndromic surveillance allows for early outbreak detection relative to traditional systems and enables community health monitoring during outbreaks ${ }^{1}$. Monitoring and disease diagnosis can be strengthened using pre-diagnostic data and statistical algorithms to detect morbidity trends.

Alerta (2002-11) and Vigila (2011-present) are sequentially implemented electronic disease surveillance systems created by the Peruvian Navy to improve the detection, prevention, and control of disease outbreaks. The phone-, internet-, and radio-based reporting system now covers over $97.5 \%$ of the Navy population ${ }^{2}$, encompassing 169 reporting establishments that treat active and retired service members, dependents, and civilian employees. Acute diarrheal disease, respiratory infections, and pneumonias are reported weekly, whereas specific notifiable diseases such as malaria, dengue, and tuberculosis are reported immediately after case detection.

\section{Methods}

Time series analysis of Alerta and Vigila reports of ADD occurring from 2009-13 were analyzed in Matlab (MathWorks vR2014a). ADD counts from each reporting location were aggregated for each epidemiological week. ADD outbreaks were retrospectively identified using Exponentially Weighted Moving Average (EMWA) control charts. Seasonal data variability was eliminated using an 8-week sliding baseline to estimate upper control limits (UCLs). UCL calculation distortion was prevented by removing outbreak week data from the baseline. A weight of $\lambda=0.3$, considered most appropriate for diseases with short incubation periods ${ }^{3}$, was used with a $k$ value of 3 standard deviations.

\section{Results}

From 2009-13, 39,764 non-bloody ADD cases were reported across all naval bases via Alerta and Vigila. Over 3\% of cases were 0-1 years old; $8 \%$ were $1-4$ years old; and $89 \%$ were over 5 years old. Non-bloody ADD prevalence tended to peak from January to March, the summer months (Fig 1). EWMA control charts detected 25 ADD outbreaks (Fig 2).

\section{Conclusions}

Real time analysis of syndromic surveillance systems such as Alerta and Vigila has the potential to promote rapid outbreak response by detecting emerging disease threats and morbidity trends in resourcelimited settings.

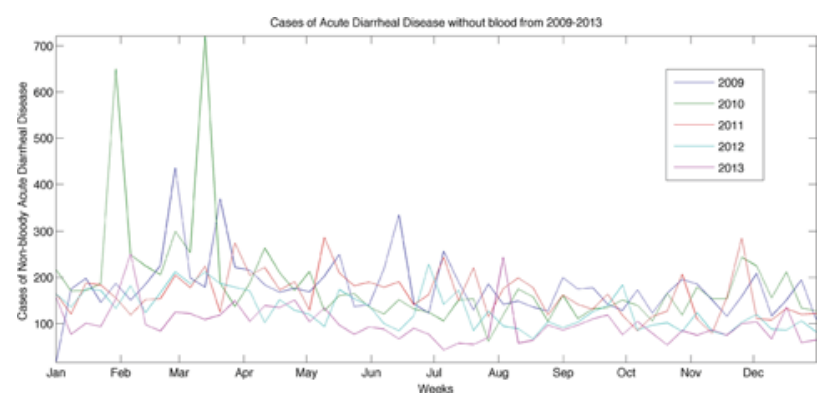

Figure 1. Cases of non-bloody ADD from 2009-2013. Outbreaks of disease were most frequent during the summer months of January to March.

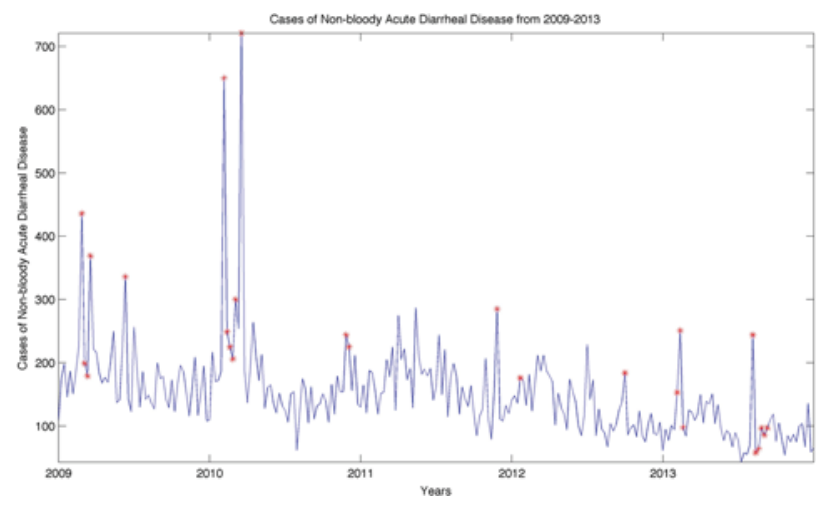

Figure 2. EWMA control charts reported 25 weeks with ADD outbreaks from 2009-2013 (denoted by asterisks).

\section{Keywords}

resource-limited setting; acute diarrheal disease; Peru; syndromic surveillance; EWMA

\section{References}

1. Jajosky RA, Groseclose SL. Evaluation of reporting timeliness of public health surveillance systems for infectious diseases. BMC Public Health. 2004 July; 4:29.

2. Mundaca CC, Moran M, Ortiz M, Saldarriaga E, Quispe J, Araujo RV, Blazes DL. Use of an electronic disease surveillance system in a remote, resource limited setting: Alerta DISAMAR in Peru. 54th Annual Meeting of the American Society of Tropical Medicine and Hygiene; 2005 Dec. 11-15; Washington, DC.

3. Buckeridge DL, Burkom H, Campbell M, Hogan WR, Moore AW. Algorithms for rapid outbreak detection: a research synthesis. J Biomed Inform. 2005 Apr; 38(2): 99-113.

\section{*Emily Alsentzer}

E-mail: ema2016@stanford.edu 